\title{
The first reported case of Kaposi's sarcoma in an AIDS patient in Sri Lanka
}

\author{
Perera H. P. ${ }^{1}$, Buddhakorale. K. ${ }^{2}$, Satgurunathan. K. ${ }^{3}$, Angunawela. P. ${ }^{4}$
}

\begin{abstract}
Kaposi's sarcoma (KS) is a rare angioproliferative neoplasm associated with human herpes virus type 8 (HHV-8) infection. Kaposi's sarcoma is an AIDS defining illness. Here we report the first case of cutaneous Kaposi's sarcoma reported in Sri Lanka in an AIDS patient in 2009.
\end{abstract}

Key words: Kaposi sarcoma, Immunosuppression, Highly active antiretroviral treatment, Sri Lanka

\author{
Authors \\ Corresponding author: ${ }^{1}$ Dr. H. P. Perera MBBS, PgDVen, MD, FSLCOSHH, Consultant Venereologist, National STD/AIDS \\ Control Programme, No 29, De Saram Place, Colombo 10, Sri Lanka, Email: pererahimali.perera@gmail.com, \\ (iD) https://orcid.org/0000-0002-4737-2763 \\ ${ }^{2}$ Dr. Kulasiri Buddhakorala, MBBS, MSC, MD, FRCP; Consultant Venereologist, Dr Neville Fernando Teaching Hospital, Sri \\ Lanka \\ ${ }^{3}$ Dr. K. Satgurunathan, Consultant Dermatologist, National Hospital of Sri Lanka \\ ${ }^{4}$ Prof. P. Angunawela, Senior Professor in Pathology, Department of Pathology, Faculty of Medicine University of Colombo \\ (c) $(7)$
}

Acknowledgement: The staff of the HIV clinic, Colombo, Conflict of interest: Authors claim no conflicts of interest Funding: No funding support for this case report, Originality, previous publications or presentations: This is an original work and there have not been any previous publications

Submitted: 23.11.2017, Accepted: 10.12.2017

\section{Full article}

Introduction

Kaposi's sarcoma (KS) is a rare angioproliferative tumour, associated with human herpes virus type 8 (HHV-8) infection (1). Four clinical variants are described

1. Classic KS (Mediterranean)

2. African endemic KS

3. Immunosuppression-associated KS

4. Post-transplant, epidemic or HIV-associated KS

All share common histological features but the clinical course is variable. Epidemic KS usually occurs in homosexual and bisexual men (3). KS may remain as an indolent cutaneous disease in patients with high $\mathrm{CD}_{4}$ counts, but in advance stages of Acquired Immune Deficiency Syndrome (AIDS) KS will disseminate (2).

During the time of the presentation of this case the estimated number of people living with HIV in Sri Lanka was 4,000 and adult HIV prevalence was $<0.1 \%$. Cumulative total of 1,059 HIV cases reported at the end of 2008 since the first identified in 1987.

\section{Case report}

A 31-year-old Sri Lankan man presented to the central sexually transmitted disease clinic, Colombo. He was deported while working in Dubai in March 2008, as he was HIV positive. He had some small, dark coloured, nodular lesions over upper and lower extremities. Lesions were painless, mildly pruritic, had increased in size during last two weeks prior to presentation. He had no other significant illness in the past. He had many unprotected 
sexual exposures with males and females, including foreigners. He was a heavy smoker and an alcoholic.

On examination, few subcutaneous hyperpigmented, non-tender plaques and nodular lesions around $0.5 \times 0.5 \mathrm{~cm}$ in size with a purplish hue were found distributed over anterior aspect of left arm and both thighs and lower part of right forearm (figure1). His oral cavity and genitalia were normal. He had no hepato-splenomegaly, lymphadenopathy or lympoedema. His eyes, palms and soles were normal. He had no neurological or gastrointestinal physical signs to suggest visceral Kaposi's sarcoma.

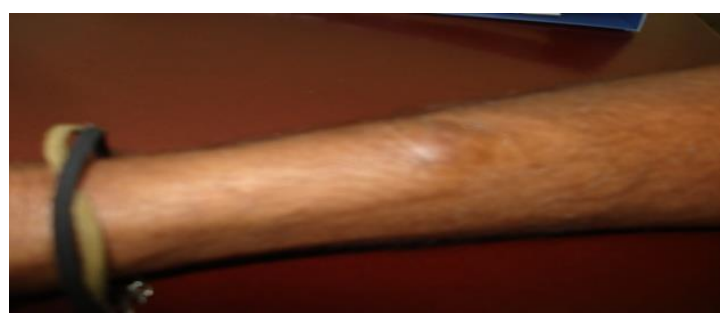

Figure1. Hyperpigmented, non-tender plaque and nodular lesions

Results of baseline investigations of the patient are mentioned in the table 1.

Table 1 Baseline investigations

\begin{tabular}{|l|l|}
\hline Investigations & Result \\
\hline VDRL,TPPA & Negative \\
\hline HBsAg, HCV Ab & Negative \\
\hline CD4, CD8 & 289,1787 cells $/ \mathrm{mm}^{2}$ \\
\hline Baseline VL & 51,044 copies $/ \mathrm{ml}$ \\
\hline CBC & Normal \\
\hline ESR & $3 \mathrm{~mm} / 1^{\text {st }}$ \\
\hline Na+, K+ & $138 \mathrm{mmol} / \mathrm{l}, 3.2 \mathrm{mmol} / \mathrm{l}$ \\
\hline S. creatinine & $0.9 \mathrm{mg} / \mathrm{dl}$ \\
\hline Blood urea & $22 \mathrm{mg} / \mathrm{dl}$ \\
\hline SGOT, SGPT & $35,28 \mathrm{IU} / \mathrm{I}$ \\
\hline Toxoplasma IgG Ab & $150 \mathrm{IU} / \mathrm{l}$ \\
\hline Mantoux, Sputum AFB & Negative \\
\hline HLAB5701 & Negative \\
\hline Chest X-ray & Normal \\
\hline Ultra Sound Scan Abdomen & Normal \\
\hline
\end{tabular}

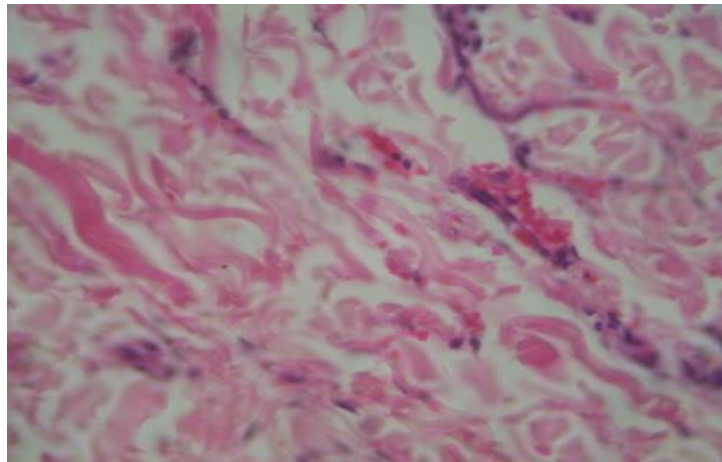

Figure2. Vascular proliferation with endothelial cells dissecting and few spindle cells.

Biopsy of a skin nodule over the arm had very early changes resembling telengiectasia, numerous vascular spaces in the reticular dermis with associated red cells. Thick collagen bundles were present in between vessels. Dilated vascular spaces were present around skin appendages. No spindle cell proliferation was seen. Biopsy was reported as 'Kaposi's sarcoma cannot be excluded'.

A second biopsy performed one month later showed dermis having vascular proliferation with endothelial cells dissecting through the dermis and skin adenexia. Few spindle cells sheets were also seen. Features were compatible with early patch stage of Kaposi sarcoma.

Patient was started on highly active antiretroviral therapy (HAART) comprising, zidovudine, lamivudine and efavirenz $(A Z T+3 T C+E F V)$ in March 2009. After eight months of treatment, AZT was substituted with stavudine (d4T) due to AZT related anaemia. Subsequently, after about one and half years of $d 4 T+3 T C+E F V$ treatment, he developed facial lipoatrophy and regimen was changed to tenofovir, emtricitabine, and efavirenz (TDF+FTC+EFV). Again, after one year of TDF+FTC+EFV treatment, the regimen was switched to abacavir, lamivudine and boosted lopinavir $(A B C+3 T C+L P V / r)$ because of virological failure of the regimen. However, he could achieve undetectable viral load after six months of $A B C+3 T C+L P V / r$ treatment and $\mathrm{KS}$ lesions disappeared completely. 


\section{Discussion}

$\mathrm{KS}$ is an AIDS defining condition. The diagnosis of KS was made in our patient based on clinical and histological evidence. At the time of the diagnosis, patient's CD4 count was 289, KS usually manifest when $\mathrm{CD}_{4}$ count is less than 200 (4). KS can occur in non-HIV individuals with other risk factors. However, this patient did not have other risk factors .Therefore, it was concluded that KS in our patient was related to HIV. It is not uncommon to have KS in HIV patients with CD4 counts over 200 (4).

Cryotherapy, laser or radiation were not required in this patient and patient responded well with the optimization of antiretroviral treatment. During one decade of follow up since the presentation, no mucocutaneous of or visceral recurrences were detected.

\section{Conclusion}

Kaposi's sarcoma should be considered in the differential diagnosis of skin nodular lesions, especially in men having sex with men with HIV infection even when $\mathrm{CD}_{4}$ Count is above 200. Optimization of antiretroviral therapy is important to avoid progression of the disease.

\section{References}

1. Foreman $\mathrm{K}$ e, Friborg J Jr, Kong W P,WoffendinC, Polverini P J, nickoloff B J, NabaelGJ,Propagation of a human herpesvirus from AIDS -associated Kaposis sarcoma. New Eng J med 1997.336:163171.

2. Haramati L B, Wong J: Intrathorasic Kaposi's sarcoma in women with AIDS, Chest 2000,117;410414.

3. Maurer T, ponateM.LeslieK,et al Kaposi's sarcoma in HIV negative men having sex with men $\mathrm{N}$ Engl J Med.2007;357(13):1352-1353.

4. Catten AM, calabro M L, De Rossi A, Aversa S MetalLong -term clinical outcome of of AIDS related Kaposi's sarcoma during highly active antiretroviral therapyInt J Oncology2005 Sep:27(3):779-85 